\title{
Modified wideband 3D late gadolinium enhancement (LGE) MRI for patients with implantable cardiac devices
}

\author{
Shams Rashid ${ }^{1 *}$, Stanislas Rapacchi ${ }^{1}$, Kalyanam Shivkumar ${ }^{2,1}$, Adam Plotnik ${ }^{1}$, Paul J Finn ${ }^{1,3}$, Peng Hu ${ }^{1,3}$ \\ From 18th Annual SCMR Scientific Sessions \\ Nice, France. 4-7 February 2015
}

\section{Background}

Late gadolinium enhancement (LGE) cardiac MRI is the clinical gold standard for non-invasive assessment of myocardial viability and plays an important role in guiding catheter ablation of ventricular tachycardia $(\mathrm{VT})^{1}$. The majority of VT patients have implanted cardiac devices such as implantable cardioverter defibrillators (ICDs). The presence of ICDs gives rise to strong off-resonance within the myocardium. This produces hyper-intensity $(\mathrm{HI})$ artifacts in LGE, which can mask scar tissue, compromising the diagnostic value of $\mathrm{LGE}^{2}$. Recent studies show that HI artifacts can be eliminated by using a wideband inversion recovery (IR) pulse in the LGE sequence ${ }^{2-3}$. However, the current wideband LGE is a $2 \mathrm{D}$ sequence, which limits spatial resolution, especially slice thickness $(8 \mathrm{~mm})$. This is problematic for using LGE to guide catheter ablation of VT. High resolution LGE is feasible using a 3D LGE sequence. However, no prior studies have explored 3D LGE under the influence of strong off-resonance imposed by ICDs.

\section{Methods}

We implemented the wideband IR pulse (bandwidth (BW) $=3.8 \mathrm{kHz}$ ) in a 3D LGE sequence (old wideband 3D LGE). Initial testing revealed extended signal void and banding artifacts, which were not present in corresponding 2D wideband LGE images. We found that these artifacts are caused by slab distortion due to off-resonance produced by the ICD. Slab distortion is inversely proportional to slab select gradient (Gss), so increasing Gss will reduce artifacts. To increase Gss, RF excitation pulse BW also needs to be increased. We modified the sequence by increasing the RF BW from $5.8 \mathrm{kHz}$ to $12 \mathrm{kHz}$ (modified wideband 3D LGE). We evaluated the new sequence in 6 volunteers (an ICD was attached to the left shoulder) and in 5 ICD patients. Image scoring (1-4 scale, $1=$ best) was used to compare artifacts between the old and modified wideband 3D LGE images in volunteers, and image quality between wideband 2D and modified wideband 3D LGE images in the ICD patients. Scores were compared using a Wilcoxon signed-rank test.

\section{Results}

Extended signal void and banding artifacts in volunteer images were significantly reduced using the modified wideband 3D LGE sequence (Fig. 1): artifact score improved from $2.7 \pm 0.6$ in the old wideband $3 \mathrm{D}$ LGE to $2.2 \pm 0.8$ in the modified wideband 3D LGE images $(\mathrm{p}<0.05)$. In the ICD patients, the modified wideband 3D LGE produced images that had higher resolution $\left(1.4 \times 1.4 \times 4 \mathrm{~mm}^{3}\right.$ vs. $\left.1.9 \times 1.4 \times 8 \mathrm{~mm}^{3}\right)$ and similar image quality scores $(\mathrm{p}=0.46)$ as wideband 2D LGE (Fig. 2).

\section{Conclusions}

We developed a modified 3D wideband LGE sequence, which reduces off-resonance slab distortion artifacts that occur in conventional 3D LGE. Our results in ICD patients yielded similar image quality and higher spatial resolution compared to wideband 2D LGE. This represents a first step towards high resolution 3D LGE imaging in ICD patients in a clinical setting.

\section{Funding}

NIH 1R21 HL118533. 

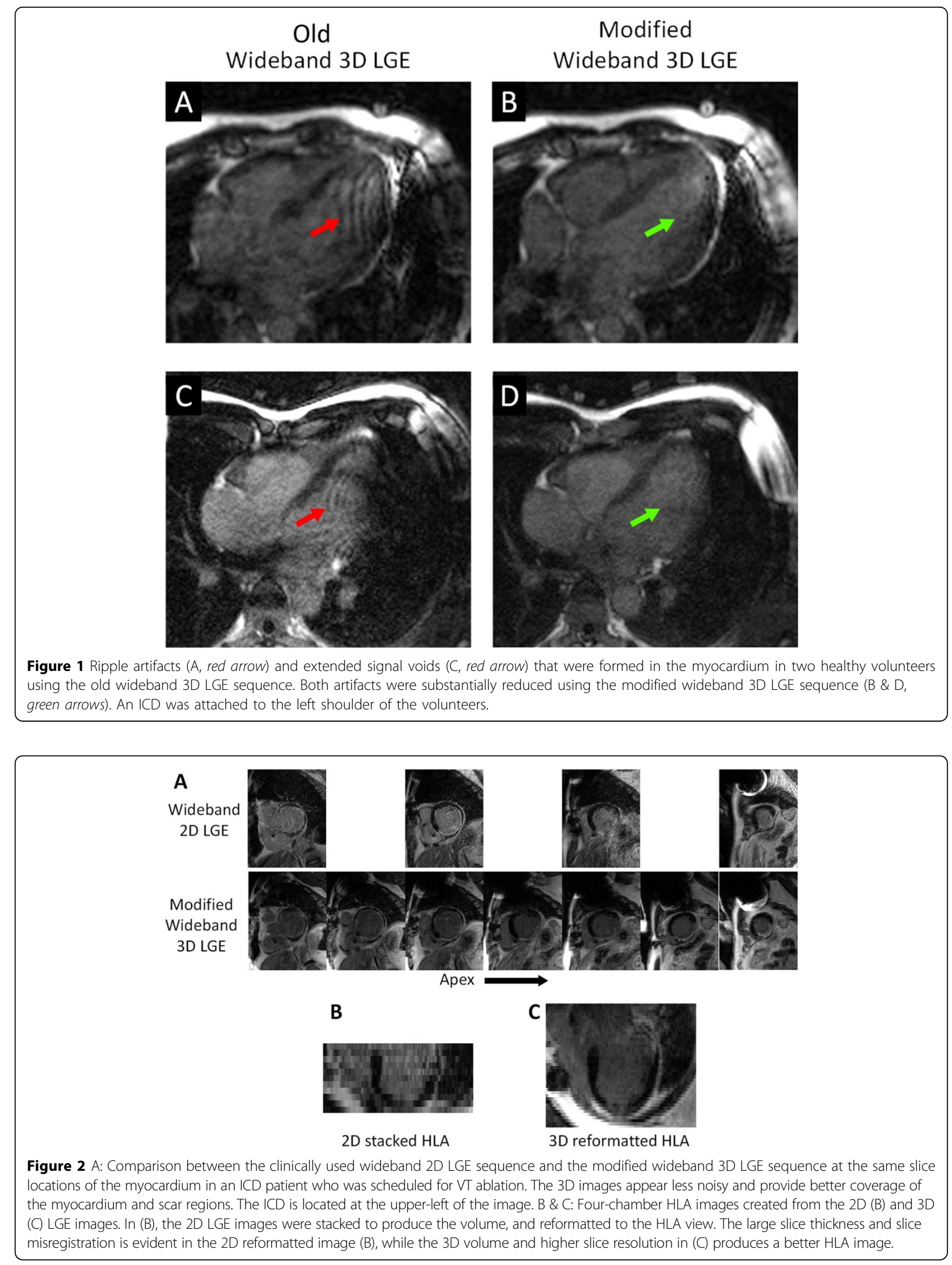


\section{Authors' details}

'Radiological Sciences, University of California, Los Angeles, Los Angeles, CA, USA. ${ }^{2}$ UCLA Cardiac Arrhythmia Center, University of California, Los Angeles, Los Angeles, CA, USA. ${ }^{3}$ Biomedical Engineering Interdepartmental Program, University of California, Los Angeles, Los Angeles, CA, USA.

Published: 3 February 2015

\section{References}

1. Circ Arrhythm Electrophysiol 2011, 4(2):172

2. Radiology 2014, 270(1):269.

3. Heart Rhythm 2014, 11(2):289.

doi:10.1186/1532-429X-17-S1-Q26

Cite this article as: Rashid et al: Modified wideband 3D late gadolinium enhancement (LGE) MRI for patients with implantable cardiac devices. Journal of Cardiovascular Magnetic Resonance 2015 17(Suppl 1):Q26.

Submit your next manuscript to BioMed Central and take full advantage of:

- Convenient online submission

- Thorough peer review

- No space constraints or color figure charges

- Immediate publication on acceptance

- Inclusion in PubMed, CAS, Scopus and Google Scholar

- Research which is freely available for redistribution

Submit your manuscript at www.biomedcentral.com/submit
() Biomed Central 